\title{
LA INFORMACIÓN CONTABLE EN LA NEGOCIACIÓN DE LOS CONVENIOS COLECTIVOS DE EMPRESA
}

\author{
Pablo Aguilar Conde \\ Basilio Cantalapiedra Nieto \\ Universidad de Burgos
}

Orcid: 0000-0001-7613-8493 y 0000-0003-3992-4764

Recibido el 30 de septiembre de 2016

DOI: $10.1387 /$ lan-harremanak. 17495

Aceptado el 20 de octubre de 2016

\section{ABSTRACT}

Resumen: La negociación colectiva se sustenta en una adecuada información económica, productiva y laboral, como condición necesaria para poder llevar a efecto el deber de negociar con buena fe, y asi facilitar el acuerdo colectivo.

En España, el Estatuto de los Trabajadores plantea la información contable a transmitir a los representantes de los trabajadores; pero esta regulación, en nuestra opinión, es tan escasa que no puede entenderse en la actual concepción de empresa como nexo de contratos y confluencia de diferentes grupos de interés.

En este contexto, el objeto de la comunicación es analizar cómo se utiliza la información contable en la negociación de los convenios colectivos de empresa en la comunidad autónoma de Castilla y León, con la pretensión de sugerir otra información económica, que de ser proporcionada a los representantes de los trabajadores, favorecería el conocimiento de la situación económica real de la empresa y consecuentemente conllevaría avances en la propia negociación. Entendemos que la información económica debe ser accesible no sólo a los representantes de los trabajadores sino a todos los trabajadores y trabajadoras de la empresa como grupos de interés internos clave de la empresa. 
Palabras clave: Información contable, negociación colectiva, retribución de los administradores

Abstract: Collective bargaining process is based on suitable economic, productive and employment information, as a necessary condition to negotiate in good faith and to sign the final agreement.

Status of Workers in Spain mentions the accounting information that the company should provide to workers; but these legal regulations, in our opinion, are so limited that cannot be taken into consideration under the new concept of company as the nexus of contracts that exist in the firm and confluence within different interest groups.

In this way, our purpose is to analyze how accounting information is used in Collective Bargaining Agreements in Castilla-León. Besides, we suggest other financial information that would facilitate a better knowledge of company economic situation and improve negotiation processes and lead to better outcomes. Economic and financial information should be accessible not only for the representatives of workers but for every worker in the company, as partner or internal key stakeholder of the company.

Keywords: Accounting information, collective bargaining agreement, remuneration of directors. 


\section{SUMARIO}

Sumario: 1. Introducción. 2. Marco teórico y revisión de la literatura. 2.1. A quién se suministra la información contable. 2.2. Qué información contable se suministra a los empleados. 2.3. Cuándo se suministra la información contable a los empleados. 2.4. Cómo reaccionan los empleados ante la información contable suministrada por la empresa. 3. Marco legal en Espańa. 4. Análisis empírico. 4.1. Objetivo y metodología. 4.2. Resultados. 4.3. Discusión de los resultados. 5. Conclusiones. 6. Bibliografía.

\section{Introducción}

La negociación colectiva desempeña un papel determinante en las relaciones laborales. El desarrollo del propio proceso negociador se agilizaría y mejoraría con una mayor y mejor transmisión de información económico-financiera.

El propósito inicial de esta comunicación es analizar a través de la revisión de la literatura específica, la transmisión de la información contable por parte de la empresa a los representantes de los trabajadores en el marco de la negociación de los convenios colectivos de empresa. Con tal motivo trataremos de responder a las siguientes preguntas:

- A quién se suministra la información contable.

- Qué información contable se suministra a los empleados.

- Cuándo se suministra la información contable a los empleados.

- Cómo reaccionan los empleados ante la información contable suministrada por la empresa.

Siguiendo este planteamiento y para constatar la realidad empresarial, realizamos una encuesta a los responsables de recursos humanos de las empresas de Castilla y León integradas dentro de la red de directores de recursos humanos de Castilla y León, CEDERED. Las conclusiones de estas encuestas las expusimos posteriormente a los sindicatos más representativos de la provincia de Burgos, mediante entrevistas que nos permitieron validar las reflexiones de la parte empresarial.

La observación y el análisis de los datos muestran que hay una preponderancia a suministrar la información mínima sugerida por el Estatuto de los Trabaja- 
dores. Sin embargo, si consideramos a los trabajadores como partícipes o stakeholders de la empresa, parecería más recomendable que la información no fuera limitativa e incluso incluyera otra información de carácter social, que facilitara el conocimiento de la política de remuneración de las empresas, tal y como se recoge en la Propuesta de Directiva del Parlamento Europeo y del Consejo de 9 de abril de 2014, por la que se modifica la Directiva 2007/36/CE en lo que respecta al fomento de la implicación a largo plazo de los accionistas y la Directiva 2013/34/UE en lo que respecta a determinados elementos de la declaración sobre gobernanza empresarial.

Todo ello, con el objetivo último de aportar, en este contexto de información y negociación colectiva, un nuevo marco de información a suministrar por parte de la empresa a los representantes de los trabajadores, que consideramos favorecería la propia negociación de los convenios colectivos de empresa.

Para conseguir el objetivo propuesto, tras este primer apartado introductorio, el segundo apartado incorpora el marco teórico en el que se sustenta el modelo de empresa que vamos a considerar e incluye una revisión de la literatura que facilite la respuesta a las preguntas formuladas. En el tercero analizamos el marco legal actual en España de la información contable en relación con la negociación colectiva y la política retributiva. En el cuarto presentamos los datos principales obtenidos tras el análisis de los cuestionarios y las entrevistas. Por último, en el quinto apartado se exponen las principales conclusiones del análisis empírico que permiten sugerir una propuesta de nuevo marco de información económica dentro de la negociación colectiva.

\section{Marco teórico y revisión de la literatura}

La Teoría de la Agencia proporciona un marco teórico útil para analizar el proceso contractual en la empresa. Define una relación de agencia todo contrato en el que al menos una parte, el agente, se compromete a realizar algo para la otra parte, el principal. La relación laboral es aquella en la cual el trabajador se compromete, a cambio de un salario, que puede o no ser función de diversos indicadores de productividad, a proporcionar un esfuerzo, productividad o rendimiento al empleador.

Las empresas incurren en costes a fin de coordinar tareas altamente interdependientes, para las cuales se requiere reunir, procesar y transmitir una gran cantidad de información, desde la alta dirección hasta los destinatarios de la misma. Los costes de negociación son los costes de transacción asociados a las negociaciones entre las partes; incluyen el tiempo empleado y los recursos gastados tanto en negociar como en mejorar la posición negociadora, así como las pérdidas soportadas por el fracaso o el retardo en conseguir un acuerdo eficiente. 
Además, también se incurre en costes de supervisión y costes para motivar a los trabajadores en quienes se ha delegado capacidad de decisión, costes de agencia. Entre ellos podemos citar los costes anteriores a la firma del contrato y negociación: costes de formalización contractual y costes de garantía, junto a los costes posteriores a esa firma/negociación: costes de control y pérdida residual. En general, son costes de las relaciones contractuales dirigidos a eliminar el conflicto entre las partes y reducir las posibilidades de incumplimiento. En definitiva, son costes de negociar y sacar adelante el contrato, en un primer momento, y posteriormente el convenio laboral; incluidos los costes de coordinación y los costes de motivación, como los que miden la actuación, crean incentivos y exigen el cumplimiento de los acuerdos para asegurar que los empleados sigan las instrucciones y cumplan los compromisos y acuerdos.

Según Jensen y Meckling (1975), la información contable puede ayudar a resolver uno de los problemas organizativos planteados: el sistema de control en su doble vertiente, evaluación del rendimiento y métodos de recompensa. Por tanto, es necesario desarrollar un sistema de control que sea consistente con la asignación de los derechos de decisión. Este sistema de control estará formado por el sistema de evaluación del rendimiento y el sistema de recompensa que alinee los intereses de los empleados con los empleadores.

El problema organizativo planteado de esta manera se centra, por tanto, en el diseño de mecanismos que asignen los derechos de decisión, midan el rendimiento y lo premien y/o castiguen. El sistema de información contable puede servir como mecanismo de información y control reduciendo la asimetría de la información (costes precontractuales) y los comportamientos oportunistas que se producen con posterioridad a la realización de los contratos (costes postcontractuales).

En este trabajo planteamos el uso de la información contable dentro de la negociación colectiva de empresa, como mecanismo de apoyo que favorece la evaluación del rendimiento de los trabajadores y que sustenta el método de retribución salarial dentro de la empresa. Los fundamentos teóricos de este trabajo se enmarcan dentro del enfoque de la nueva economía institucional, en el cual la empresa es aquella organización compleja que opera en situación de incertidumbre e información limitada, donde surgen problemas derivados de la ausencia de información perfecta.

De entre los diferentes contratos que configuran la empresa, nos centramos en el contrato de empleo. Éste se desarrolla en un marco teórico de asimetría informativa, incertidumbre, racionalidad limitada y oportunismo, donde la relación empleador-empleado puede caracterizarse como un contrato incompleto, bilateral y con relación de autoridad. Se trata de la Teoría de los contratos que recientemente ha tenido mayor repercusión mediática al ser premiados con el Nobel de Economía 2016 sus mentores Oliver Hart y Bengt Holmström. Am- 
bos economistas «han desarrollado la teoria de los contratos, un amplio marco de análisis de los múltiples aspectos del contrato, como la remuneración de los directivos basada en sus resultados,...», explicó Hansson, secretario general de la Real Academia, en el anuncio del premio Nobel de Economía. El economista finlandés Holmström, por su parte, se interesó en los contratos de trabajo, y especialmente en los temas de remuneración, desarrollando tres factores que entran en juego: "la asimetría de información, las incertidumbres y las divergencias de intereses». "Gracias a la investigación de Oliver Hart y Bengt Holmström, tenemos ahora los instrumentos para analizar no solamente los términos financieros de los contratos, sino también la prestación contractual de los derechos de control, de los derechos de propiedad y de los derechos de decisión entre las partes», explicó la Academia Real de Ciencias de Suecia en el comunicado del Nobel de Economía 2016.

El origen del estudio del tema relativo a información contable y negociación colectiva podemos situarlo en el trabajo de Pillsbury «Organized labor's view of corporate financial information" publicado en 1958. Su desarrollo posterior tuvo su momento culminante entre los años 1980-1990, y sobre todo en Alemania, Estados Unidos, Gran Bretańa y los países escandinavos. En España, sin embargo, apenas hay estudios sobre el tema, si exceptuamos los trabajos de Pons (1996) y Laffarga (1993).

$\mathrm{Al}$ inicio de los 90, el tema queda estancado al ser tratado por otras disciplinas y con otro enfoque, dentro del concepto más amplio de la responsabilidad social corporativa que últimamente se pretende divulgar mediante el informe integrado. Gran parte de la concepción actual de la información contable para los empleados está subyacente en las diferentes posturas de las empresas hacia la responsabilidad social, donde los empleados son un grupo de interés interno a considerar, en igual medida que lo son los socios y accionistas. Así, a partir del año 2000 no ha habido nuevas aportaciones relevantes relativas a la información contable para la negociación colectiva, a pesar de que las circunstancias económicas desfavorables parece que sugieren una mayor relación con los planteamientos necesarios en las revisiones salariales de los convenios colectivos de las empresas.

\subsection{A quién se suministra la información contable}

En el análisis de la utilidad de la información contable también se identifica a los trabajadores como usuarios de la información contable, indicando que esta información interesa a los mismos y a sus representantes, ya que como estamento base de la producción estarán interesados en los resultados obtenidos por las organizaciones para la revisión de los convenios, la negociación de las condiciones de trabajo, la propia continuidad del empleo, etc.

Sin embargo, la información que utilizan los sindicatos y los representantes de los trabajadores no tiene porqué coincidir con la utilizada por los diferen- 
tes tipos de usuarios. Craft (1981) destaca que hay diferentes factores dentro de cada empresa y cada organización sindical, que pueden alterar el contenido de la información contable útil para cada sindicato. Para Amernic y Craig (1992) hay dos factores que inciden fundamentalmente en el uso que se hace de la información contable: la ambigüedad de la parte empresarial (employer equivocality) y la heterogeneidad sindical (union heterogeneity). Para estos autores, cuando la ambigüedad empresarial es baja y la heterogeneidad sindical alta, la contabilidad puede servir como un primer punto para la discusión.

Podemos clasificar las diferentes líneas de investigación detectadas en la revisión de la literatura en dos grupos: aquellos que consideran que todos los trabajadores deben ser receptores de la información frente a los que consideran que la información contable sólo debe ser transmitida a los representantes de los trabajadores.

Existen diferentes maneras de usar esa información contable dependiendo de varios factores (Rahman y McCosh, 1976: 343-350). Los usuarios más importantes (high-use style) centran su importancia en la evaluación del comportamiento y toma de decisiones. Los usuarios menos importantes (low-use style) consideran la información contable relativamente insignificante, prestando más atención a las relaciones interpersonales. Los usuarios medios (medium-use style) consideran tanto a la información contable como a las relaciones interpersonales. Podríamos considerar a los trabajadores dentro de este último tipo de usuarios, puesto que utilizan tanto la información contable, como cualquier otra que puedan conseguir por otros medios.

Se produce un crecimiento de demanda de información contable por parte de los representantes sindicales, al entender que la posesión de esta información permite afrontar la negociación desde una mejor posición que ante la ausencia de información económico financiera. En el entorno de conflictividad laboral, la posesión de más y mejor información por una de las partes permite enfrentarse a la negociación con terceros desde una situación de poder (Del Brío González, 1995: 801-818). La asimetría informativa es fuente de situaciones de desequilibrio y se hace necesario asumir el coste de la información si el beneficio esperado con la negociación supera ese coste. Los empleados quieren tener acceso a la información contable para mejorar su posición negociadora y los empleadores deben suministrar esa información porque el beneficio que pueden obtener con una negociación ágil y racional (menor conflictividad social y mayor productividad de los trabajadores), superará los costes de transmitir esa información.

La información contable puede ser aceptada por los trabajadores si facilita que los empleados tomen decisiones, y siempre que se constate que mediante su utilización se mejora el bienestar o la situación de los trabajadores de cada empresa (Cooper y Essex, 1977: 215-216). En la medida en que los diferen- 
tes grupos de personas relacionados con la empresa son usuarios de la información contable, no se puede entender por qué casi nunca se considera a los trabajadores en el momento de confeccionar esta información (Cooper y Sherer, 1984: 212-215), si después de todo, los intereses de los trabajadores no van a ser independientes de los de otros grupos.

Si la empresa debe suministrar información contable a los representantes de los trabajadores nos faltaría determinar el nivel de agregación de esa información: nacional, sectorial, o de empresa. Así, limitaremos el tema centrándonos en la información a nivel de empresa y la posible utilidad de esta información para la toma de decisiones como, por ejemplo, las decisiones relacionadas con el incremento salarial y todas aquellas otras que constituyen el contenido de los convenios colectivos de empresa.

Dentro de los diferentes trabajadores, se diferencia entre trabajadores de cuello azul y de cuello blanco, entre miembros del comité de empresa o miembros sindicales, y entre representantes a tiempo parcial o de tiempo completo (Jackson-Cox, Mcqueeny y Thirkell, 1984: 265-268). Owen y Lloyd (1985) distinguen el uso de la información contable dependiendo de la naturaleza de la organización, es decir, de la empresa. Así, para estos autores la importancia de la información depende de los objetivos y de la cultura de la empresa y de los sindicatos.

Por lo tanto, se preparan informes destinados a los accionistas, a la dirección, a los acreedores y por qué no, para la negociación empleador - empleado. Hasta ahora, parece que se ha primado el uso de la información contable por parte de los accionistas y de los acreedores. Sin embargo, los trabajadores pueden ser el motor de la empresa, dentro de la idea de empresa entendida como un nexo de contratos. De hecho, éstos demandan cada vez más información, incluida la contable. El único punto de discusión podría ser determinar si los trabajadores son usuarios internos o externos de esa información contable.

\subsection{Qué información contable se suministra a los empleados}

Las diferentes posturas encontradas en la revisión de la literatura abarcan desde los que consideran que la información contable a transmitir debe ser únicamente la mínima requerida, hasta los que defienden la total transmisión de información, atendiendo siempre a los requerimientos por parte de los representantes de los trabajadores. No todas las empresas suministran el mismo tipo de información. Según Jackson-Cox, Thirkell y McQueeney (1984) podemos encontrar dos tipos de empresas: aquellas que suministran la información como una rutina (the integrated approach) y aquellas otras que sólo dejan acceso a la información específica en determinadas situaciones (ad-hoc approach). 
La información que generalmente se transmite a los empleados es la referente a salario base, productividad, condiciones de trabajo, despidos, condiciones de empleo, seguridad en el trabajo y condiciones a largo plazo (Sherer, Soutworth y Turley, 1981: 8-11). Según estos autores, toda esta información es considerada insuficiente por parte de los empleados. Además, se nota un diferente grado de información por parte de las empresas dependiendo del tamaño. Así las pequeñas empresas son las que menos informan. Todo esto puede ser debido a que en las pequeñas empresas no existe una separación rígida entre la propiedad y la dirección, mientras que la separación en las grandes empresas es radical y se requiere más información por parte de los diferentes usuarios.

El uso e incluso la petición de esta información son también distintos por parte de los trabajadores de "cuello blanco» y de "cuello azul». El uso de la información depende de la naturaleza de la representación sindical (Jackson-Co et al., 1984: 270). Según Pacitti (1990), la información que tiene por objetivo implicar a los individuos en particular, debería ser distinta de la información que se transmite a los representantes de los trabajadores y que utilizarán en la negociación colectiva. Sin embargo, el derecho a recibir la información es igual para todos los empleados. Nos encontramos, otra vez, con la información asimétrica de la situación económica de la empresa y el poco interés en cuanto a la transmisión de esa información por parte del principal o parte empleadora en la empresa. Los empleados no pueden obtener toda la información deseada y tampoco pueden controlar el proceso de su elaboración.

Para Gospel (1978) podemos encontrar tres posturas en lo referente a la transmisión de la información contable a los empleados. La postura del avestruz, the ostrich approach, en la que hay una falta de interés por los datos de la empresa; postura claramente rechazable. La postura de información mínima o shopping list. La postura de información de interés razonable para los empleados, the decisión orientated approach. El segundo planteamiento podría ser un punto de partida hasta llegar a la última posición.

Siguiendo a Ogden y Bougen (1985), el deseo de recibir información por parte de los empleados es una postura que se remonta hacia el concepto marxista de lucha de clases entre trabajo y capital; debido al conflicto de intereses se valora el mayor contenido y calidad de la información contable. Los empleadores quieren ser capaces de usar a los trabajadores de la manera más flexible posible, tratando de minimizar su coste; mientras que los empleados quieren ejercer algún control sobre la labor de los empleadores, para de alguna manera minimizar su inseguridad y explotación. Así, según la opinión de estos autores, los trabajadores van a demandar diferentes informaciones, que gradualmente pueden resumirse de la siguiente manera: toda la información o sólo información en determinadas situaciones. Desde las empresas, se revelará información 
siempre y cuando el beneficio esperado supere al coste de preparar y proporcionar esa información.

La transmisión de información a los empleados puede llevarse a cabo bajo dos modalidades no excluyentes. Información particularizada en cada empleado (role related information) e información generalizada al conjunto de los empleados (employee reporting) (Maunders, 1981: 2-5). Dejando al margen la información del primer tipo y centrándonos en la segunda modalidad podemos conseguir los siguientes objetivos: informar a los empleados, influir directamente a los empleados; e influir a los empleados a través de otras personas (sus representantes). La información puede ser decisiva en el campo motivacional en el siguiente aspecto: cuanto más control se ejerce sobre los empleados, menos confianza se tiene sobre la labor de los directivos (Fox, 1974), por tal razón, la información trata de devolver esa confianza a pesar del control.

Para Walton y Mckersie (1965), la influencia ejercida por la información no es uniforme ya que la negociación presenta diferentes actividades: Distributive bargaining tiene como función resolver puros conflictos de intereses, representa juegos de suma fija; Integrated bargaining trata de encontrar intereses complementarios y solucionar los problemas con un beneficio compartido, según la teoría de juegos de suma variable; Attitudinal structuring, cuya misión es influir la actitud de la contraparte; Intraorganisational bargaining, tratando de encontrar el consenso entre los diferentes grupos que participan directamente o indirectamente en la negociación, es decir, alinear expectativas del agente con las del principal (Tracy y Peterson, 1986: 95-96). Pues bien, dependiendo en qué grado de la negociación estemos en cada situación, el contenido de la información contable se verá modificado.

Los problemas que se deben solucionar en lo referente a la información contable que se debe revelar para la negociación colectiva, surgen como consecuencia de la contraposición de intereses entre empleadores y empleados (Barbash, 1979: 648-652). La naturaleza adversa de estos grupos que componen la empresa nos puede llevar, mediante el análisis de los diferentes objetivos de cada parte, a plantear los diferentes factores que inciden en la transmisión de la información contable (Craft, 1981: 99-102). Según este autor, el primer factor sería el poder de negociación: a medida que es mayor para la dirección disminuye la necesidad de suministrar esa información contable. En segundo lugar, la independencia de la empresa. Si la empresa aplica el convenio del sector, no tiene demasiados incentivos para aumentar la transmisión de información. En tercer lugar, la conflictividad. Si existe una buena sintonía con la organización sindical se suele tender a dar más información que la mínima legal, porque incluso este hecho hace mejorar la cooperación entre las dos partes. En cuarto lugar, la estabilidad del sindicato. Si dentro de los representantes de los trabajadores hay un liderazgo y no hay facciones discordantes se evita la conflictividad y estaríamos 
en la situación planteada por el tercer factor. En quinto y último lugar, Craft cita la formación sindical que puede facilitar la comprensión y la discusión de todo tipo de información, incluso la contable.

Pero esa información que se puede o debe transmitir a los empleados, ¿debe ser exclusivamente preparada para ellos o es la generalmente utilizada por el resto de los usuarios? La transmisión de la misma información puede llevar a su incomprensión y quizás a querer participar en la cifra de beneficios, que en algunas ocasiones pudiera parecer excesivamente elevada; para Pacitti (1990), se deberían elaborar unos estados más simplificados y especialmente destinados a la comprensión por parte de los empleados de la empresa. No debemos olvidar que el problema de la revelación de información depende de la cantidad y del tipo de información proporcionada. Así, para Cuthbert y Whitaker (1977) a nivel de empresa o de planta industrial se puede producir más transmisión de información. El único problema que podríamos discutir sería si esta información a nivel de planta es para todos los empleados o únicamente para los representantes de estos trabajadores.

La contabilidad afecta al comportamiento humano (Amernic, 1983: 28-30), facilita la toma de decisiones, y como tal debería jugar un mayor papel en la negociación colectiva. La información contable ha sido introducida por diferentes autores como elemento que facilita la resolución del problema de incentivos, debido a la información asimétrica, la existencia de contratos incompletos, racionalidad limitada y el comportamiento oportunista como consecuencia de la divergencia de intereses entre la actuación del agente y el objetivo del principal. Pero el tipo de información contable transmitida a los empleados, sindicatos o representantes de los trabajadores depende de la situación por la que atraviesa la empresa. Las opiniones mayoritarias indican que la empresa tiende a informar para dejar entrever o demostrar su incapacidad de pago, es decir, evidenciar su incapacidad para afrontar una subida salarial.

\subsection{Cuándo se suministra la información contable a los empleados}

El hecho que la información contenida en las cuentas anuales sea comprensible, relevante, comparable, oportuna y sobre todo fiable, facilita el uso de la misma en la renegociación de los contratos. La fiabilidad u objetividad como punto central de discusión queda demostrada en la literatura cuando se plantea, incluso ante los tribunales, la capacidad para pagar de las empresas (Ability To Pay). De nuevo, estamos ante información asimétrica, en este caso completo conocimiento por parte de la dirección o principal en términos relación empleador - empleado y casi total desconocimiento, o por lo menos dudas sobre su exactitud, por parte de los trabajadores o agentes de la relación. La capacidad de pago de las empresas es un asunto muy tratado en las negociaciones colectivas pero es algo que admite diferentes criterios. Si la información contable se mani- 
pula puede ser debido a que realmente sí tiene impacto en las subidas salariales, y es esta razón por la que se dedica tanto esfuerzo en esconder la realidad económica de las empresas (Yamaji, 1986: 13-15).

Sin embargo, puede que no sólo sea manipulación sino que la información contable, no es el único dato que se debe tener en cuenta para fijar la incapacidad o capacidad de pago (Amernic y Craig, 1992: 63-66). Según estos autores, los representantes de los trabajadores encuentran unas limitaciones al uso de la información contable: falta de información futura, demora en su transmisión, énfasis en la información dirigida a los accionistas, irrelevancia.

El momento en el que se transmite más información contable y más frecuentemente es durante e inmediatamente antes de las huelgas (Gibbins, Richardson y Waterhouse, 1990: 123). Debido a que las huelgas tienen un coste elevado, tanto a corto como a largo plazo, planteamos que la huelga podría ser evitada si se transmitiera información periódicamente y no sólo debido a la conflictividad laboral. Si se informa durante las huelgas es para resolver la conflictividad laboral y favorecer un acuerdo; así, si se informara periódicamente trataríamos de prevenir la conflictividad y evitar llegar a la huelga, disminuyendo ese coste de la renegociación bajo presión sindical. Por otra parte, si se informa durante las negociaciones es debido a que esta información es relevante, lo que puede indicar que existe alguna relación entre la información contable y la negociación colectiva.

Bajo la perspectiva de Ogden (1992) la transmisión de información contable a los trabajadores implicaría que éstos quisieran incluso participar en la gestión y organización de la empresa, ante lo cual se plantea un fuerte rechazo por parte de las organizaciones empresariales. Además, los empresarios se reafirman en la necesidad de no suministrar información contable a los empleados, ya que de lo contrario, si dispusieran de información de la empresa, podrían efectuar alguna medida de presión, como por ejemplo paradas de producción o huelgas, en el momento más inoportuno para la empresa. En definitiva, según este autor, el beneficio potencial procedente de la transmisión y cooperación de los trabajadores, se podría volver en un coste de mucha mayor magnitud para la empresa.

La transmisión de la información contable es diferente según cuatro factores socioeconómicos (Lewis et al., 1984: 277-278). Estos factores son: las nuevas tecnologías, el sector industrial, la presión sindical y la recesión económica. Según estos autores, siempre que hay un periodo de gran interés por la información, posteriormente va seguido de un plazo de estancamiento o escaso interés por la misma.

Generalmente la información contable se revela a los empleados, sindicatos y representantes de los trabajadores como consecuencia de la política de direc- 
ción de la empresa y casi nunca como respuesta a peticiones sindicales (Mitchell et al., 1980: 55-56).

Para el empleador, existen diferentes costes como consecuencia de preparar esa información y transmitirla a los empleados. Así, que en principio sólo suministran la información contable cuando el coste de la obtención y preparación de esa información es superado por los beneficios con posibilidades de consecución durante la negociación colectiva (Kleiner, 1984: 255). Lo que generalmente sucede cuando tratan de evidenciar la incapacidad de pago. Por parte del empleado, sólo se utilizará la información contable cuando los beneficios que obtengan superen los costes de su utilización (Sherer et al., 1981: 6-11).

Los beneficios son privados y las pérdidas son públicas. Esta es la frase que resume la tendencia general que se deduce de la revisión de la literatura y que sitúa principalmente la transmisión de información contable ante circunstancias económicas negativas, no así ante situaciones positivas.

\subsection{Cómo reaccionan los empleados ante la información contable suministrada por la empresa}

A pesar de que los empleados reclaman esta información contable, la mayor parte de los autores analizados muestran las dudas que tienen los trabajadores ante la realidad de tal información, temiendo en la mayor parte de los casos la manipulación contable.

Además, no todas las empresas tienen el mismo carácter y tipo de actividad, con lo que la diferencia de utilización de la información contable se hace más palpable. Así, por ejemplo, Amernic (1985) plantea, mediante el método del caso, la utilización de la contabilidad para la negociación en una gran universidad canadiense. La diferente disponibilidad de los datos financiero - contables se puede apreciar a lo largo de toda la literatura revisada. Por un lado, la parte sindical expone la falta de información que queda en sus manos; mientras que la empresa razona esa escasez de información, escudándose en la protección integral de los datos de la empresa. Así pues, nos encontramos dentro del marco conceptual de partida, la existencia de información asimétrica.

La forma de utilizar esa información asimétrica por parte de la dirección y los sindicatos se analiza por la teoría de la agencia (Salamon y Smith, 1979: 320). Unimos dos ideas: información asimétrica y maximización de los propios beneficios. En base a esas dos ideas trataremos de analizar la reacción de empleados y empleadores. Los empleados tienden a disminuir sus pretensiones a medida que transcurre el conflicto salarial. Los empleadores tienden a aumentar las concesiones a medida que se desarrolla el conflicto. Según Hicks (1963), 
el salario de equilibrio será aquel que concilie las dos posturas. No obstante, las posturas de ambas partes, se pueden desplazar según sea el grado de información disponible para cada parte, como por ejemplo la duración de la huelga prevista o la incapacidad de pago por parte empresarial. Así, por ejemplo, el empleador es indiferente entre el incremento salarial y una cierta duración de la huelga si el valor de los costes de la huelga es coincidente con la nueva petición salarial (Yamaji, 1986: 15-16).

La receptividad de la información contable por parte de los empleados no siempre es total. Por un lado los empleados encuentran demasiado agregada la información que se les suministra y también presumen la posible manipulación de los datos para afrontar la negociación. Existen multitud de estudios sobre la diferente elección del método contable en fechas anteriores, próximas o durante la negociación (Tomczyk, 1975).

Liberty y Zimmerman, (1986) examinan la hipótesis de que los empleadores reducen (manipulan) las ganancias durante las negociaciones; hipótesis rechazada, ya que se puede deshacer la manipulación por parte sindical. En el otro sentido, Culliman, Clark y Kniblett (1994) analizan las elecciones contables referentes a la amortización y la valoración de las existencias según el grado de sindicación existente en la empresa. Así, mediante el uso de variables dummy contrastan la hipótesis siguiente: la existencia de mayor sindicación lleva aparejada un mayor maquillaje de los estados contables, con el objetivo de tener argumentos en contra de las subidas salariales, propuestas por los representantes de los trabajadores. Hipótesis que queda contrastada y que sobretodo es más evidente en empresas de algún sector específico. Al fin y al cabo, lo que tratan de demostrar las empresas es su incapacidad o limitación para hacer frente a nuevas subidas salariales.

En la misma línea, Yamaji (1986) contrastó cómo las empresas que cierran sus cuentas en noviembre y diciembre son más "pesimistas" que aquellas que lo hacen en marzo, por una razón fundamentalmente: las que cierran en diciembre están próximas al proceso negociador, mientras que cuando lo hacen en marzo, tiene lugar después del proceso negociador.

Los diferentes factores y características de la relación empleador - empleado junto al grado de utilidad de la información para el sindicato nos llevan a delimitar las diferentes posturas de la relación empleador - empleado: desde conflicto permanente con poca información y poca utilización por parte sindical, hasta cooperación con mucha información y utilización por parte de los representantes de los trabajadores.

La reacción de los empleados es de aceptación o de rechazo. Así, quienes aceptan la información contable es porque la toman como información veraz y objetiva. Knights y Collinson (1987) concluyen que los trabajadores ven 
la información contable como objetiva, incluso cuando demuestra situaciones de insolvencia o de grandes pérdidas; los que más aceptan esta información son los hombres, trabajadores de cuello azul, según estos autores. Sin embargo, a medida que los trabajadores se van formando se tiende a rechazar esta información, al entenderla como información de carácter subjetivo (Bougen, 1989).

Así, por ejemplo, para Walton y Mckersie (1965), dentro de la negociación típica distributive bargaining, la reacción de los empleados frente a la información contable es variada. Para Craft (1981) el hecho de transmitir información otorga poder a la otra parte (los empleados) y aumentan las peticiones, con lo que recomienda no revelar información. Por otra parte, Peel y Pope (1981) consideran que es mejor transmitir esa información ya que disminuyen las expectativas de los trabajadores; si después de todo, los empleados desarrollan expectativas racionales y moderarán sus peticiones antes circunstancias económicas negativas.

Oakes y Covaleski (1994) examinan la reacción de los empleados ante las medidas de control por parte de los empleadores. Así, concluyen que los empleados tienden a rechazar las medidas técnicas como análisis de métodos y tiempos, mientras que se muestran a favor de los resultados contables como mecanismo para evaluar su actuación. Una vez analizado el rendimiento plantean dos mecanismos para fijar las recompensas: repartir el ahorro del coste o repartir los beneficios.

Como dato significativo que puede, en algunas ocasiones, demostrar que la reacción de los empleados ante el suministro de la información contable es positiva, podríamos destacar el hecho de que el número de días perdidos como consecuencia de huelgas es mucho menor en Alemania y Japón que en Estados Unidos. Es en Alemania y en Japón, donde se transmite más información contable a los empleados, sindicatos y representantes de los trabajadores (Kleiner, 1984: 255).

Si como hemos visto no siempre los empleados disponen ni del mismo contenido de información contable, ni tienen acceso a ésta en el mismo horizonte temporal, nos debemos plantear cómo reaccionan los empleados ante esta información. Problemas como la falta de fiabilidad e información oportunista constituyen el elemento común en este punto. Por el contrario, si el contable fuera visto como objetivo e independiente, podría ser el intermediario entre las dos partes contrapuestas: empleador y empleado (Goggans, 1964: 628).

Todo ello, en la línea desarrollada por Hopwood (1985), de la importancia de la información contable en su relación con la sociedad y los aspectos sociales. Se pretende convertir lo social en económico y lo económico en social. 


\section{Marco legal en España}

Según los arts. 87 y 88 del Estatuto de los Trabajadores (ET, 2015)리 las partes legitimadas para negociar los convenios colectivos marco son los sindicatos y asociaciones empresariales "que cuenten con la legitimación necesaria, de conformidad con lo establecido en la presente Ley». Las partes negociadoras en los convenios colectivos empresariales o de ámbito inferior son:

a) Del lado de los trabajadores, alternativamente y no acumulativamente (Sentencia del Tribunal Supremo de 14 de julio de 2000), bien la representación unitaria (el comité de empresa o los delegados de personal, en su caso), bien la representación sindical (las secciones sindicales de los sindicatos más representativos a nivel estatal o de Comunidad Autónoma y de los que tengan representación en el comité de empresa o en los delegados de personal: art. 8.2.b) de la Ley Orgánica de Libertad Sindical) que, en su conjunto, sumen la mayoría de los miembros del comité de empresa (art. 87.1 del ET).

b) Del lado empresarial, el empresario o sus representantes (art. $88.1 \mathrm{del}$ ET). Una asociación empresarial no podrá negociar a este nivel, salvo representando voluntariamente a un empresario.

Las partes negociadoras son libres para determinar el ámbito de aplicación funcional (intersectorial, sectorial, subsectorial, grupo de empresas, empresarial o de centro de trabajo) territorial (estatal, interprovincial, de Comunidad Autónoma, provincial, comarcal o local) y personal (colectivos o grupos de trabajadores) de un convenio colectivo (art. 83.1 del ET).

El convenio colectivo puede negociarse para todos los trabajadores de la empresa o parte de ellos, existiendo en principio libertad para negociar por separado determinados grupos de trabajadores de una empresa, siempre que no se atente contra el principio de igualdad de trato, debiendo tener una justificación objetiva y razonable (arts. 14 CE y 17 ET; Sentencia del Tribunal Constitucional 136/1987, de 22 de julio).

El derecho de información viene expresamente regulado en el ET:

Art. 64.1: "El comité de empresa tendrá derecho a ser informado y consultado por el empresario sobre aquellas cuestiones que puedan afectar a los trabajadores, asi como sobre la situación de la empresa y la evolución del empleo en la misma, en los términos previstos en este articulo»

1 El Real Decreto Legislativo 2/2015, de 23 de octubre, por el que se aprueba el texto refundido de la Ley del Estatuto de los Trabajadores vigente en la actualidad deroga el Real Decreto Legislativo 1/1995, de 24 de marzo, por el que se aprueba el texto refundido de la Ley del Estatuto de los Trabajadores y otras disposiciones relacionadas, para actualizar su contenido. 
"En la definición o aplicación de los procedimientos de información y consulta, el empresario y el comité de empresa actuarán con espiritu de cooperación, en cumplimiento de sus derechos y obligaciones reciprocas, teniendo en cuenta tanto los intereses de la empresa como los de los trabajadores»...

Art. 64.2: "El comité de empresa tendrá derecho a ser informado trimestralmente:

a) Sobre la evolución general del sector económico a que pertenece la empresa.

b) Sobre la situación económica de la empresa y la evolución reciente y probable de sus actividades, incluidas las actuaciones medioambientales que tengan repercusión directa en el empleo, asi como sobre la producción y ventas, incluido el programa de producción»...

Art. 64.4: "El comité de empresa, con la periodicidad que proceda en cada caso, tendrá derecho a:

Conocer el balance, la cuenta de resultados, la memoria y, en el caso de que la empresa, prevista la forma de sociedad por acciones o participaciones, los demás documentos que se den a conocer a los socios, y en las mismas condiciones que a éstos»...

Art. 64.6: "La información se deberá facilitar por el empresario al comité de empresa, sin perjuicio de lo establecido específicamente en cada caso, en un momento, de una manera y con un contenido apropiados, que permitan a los representantes de los trabajadores proceder a su examen adecuado y preparar, en su caso, la consulta y el informe»...

En definitiva, el comité de empresa tiene derecho a ser informado sobre la situación de la empresa en los términos previstos en este artículo 64 del Estatuto de los Trabajadores. Así, parece que la información a suministrar es la expresamente recogida en este artículo.

Sin embargo, las posteriores referencias al espíritu de cooperación y la expresa literalidad "los demás documentos que se den a conocer a los socios» deja abierta la posibilidad de ampliar la información en cada caso particular, a requerimiento razonado de los propios representantes de los trabajadores "de una manera y con un contenido apropiados»...

Uno de los aspectos tratados en la negociación del convenio colectivo de empresa es el que tiene que ver con la retribución de los trabajadores. Consideramos, en línea con la Propuesta ${ }^{2}$ de Directiva del Parlamento Europeo y del Consejo de 9 de abril de 2014, por la que se modifica la Directiva 2007/36/CE en lo que respecta al fomento de la implicación a largo plazo de los accionistas y la Directiva 2013/34/UE en lo que respecta a determinados elementos de la declaración sobre gobernanza empresarial, que la información suministrada a los representantes de los trabajadores debiera incorporar los siguientes aspectos recogidos expresamente en dicha Propuesta de Directiva. Oficial.

2 Propuesta de Directiva que, a 28 de noviembre de 2016, no está publicada aún en el Diario 
En la exposición de motivos de dicha Propuesta de Directiva se recalca que los accionistas deben controlar las estrategias de remuneración de los consejeros, precisando las modalidades de información para los accionistas sobre dichas remuneraciones:

"La información revelada por las sociedades no es completa, clara ni comparable; en segundo lugar, los accionistas a menudo carecen de vías para expresar su opinión sobre la remuneración de los consejeros»

"Con el fin de garantizar la rendición de cuentas de los consejeros, dicho informe debe ser claro y comprensible e incluir una descripción completa de la remuneración percibida por cada consejero en el último ejercicio"

"Para que los accionistas puedan acceder con facilidad a toda la información pertinente sobre la gobernanza empresarial, el informe relativo a las remuneraciones debe formar parte de la declaración sobre gobernanza empresarial que las sociedades cotizadas deben publicar de conformidad con el artículo 20 de la Directiva 2013/34/UE del Parlamento Europeo y del Consejo, de 26 de junio de 2013»...

Por tanto, si tal y como apuntaba el artículo 64.4 del ET, los representantes de los trabajadores tienen derecho a recibir la información suministrada a los socios, también podría entrar dicha información.

Por otro lado, lo recogido en el artículo 9 bis y ter de la citada Propuesta de Directiva sería sumamente interesante para facilitar la negociación colectiva, en garantía de la equidad y la justa distribución del valor generado por la compañía.

"...explicando la ratio entre la remuneración media de los consejeros y la remuneración media de los trabajadores a tiempo completo de la sociedad distintos de los consejeros, y los motivos por los que esa ratio se considera apropiada»

Es obvio que los representantes de los trabajadores estarían totalmente interesados, no tanto en conocer la remuneración de los administradores, sino la proporcionalidad en relación con la remuneración de los trabajadores. Esta información sí sería un ejercicio de transparencia, que facilitaría elementos objetivos suficientes a la hora de plantear la variación de la retribución salarial dentro del convenio colectivo de empresa.

Articulo 9 ter

"... que la sociedad elabore un informe sobre las remuneraciones claro y comprensible, que ofrezca una descripción detallada de la remuneración —incluidas todas las prestaciones en cualquiera de sus formas - concedida durante el último ejercicio a cada consejero, incluidos los antiguos consejeros y los contratados recientemente. Contendrá, cuando proceda, todos los elementos siguientes:

a) la remuneración total concedida o abonada, desglosada en sus componentes, la proporción relativa de la remuneración fija y variable, una explicación de la forma en que se vincula la remuneración total con el desempeño a largo plazo, e información sobre la manera en que se han aplicado los criterios de desempeño. 
b) la variación relativa de la remuneración de los consejeros a lo largo de los tres últimos ejercicios y su relación con la evolución del valor de la sociedad y con la variación de la remuneración media de los trabajadores a tiempo completo de la sociedad distintos de los consejeros"...

En definitiva, consideramos que bajo la perspectiva de la teoría de los stakeholders, donde tanto los administradores como los trabajadores son grupos de interés internos de la empresa, debería mejorarse la transmisión de información, para que la, en teoría, «responsabilidad social de la empresa», quede demostrada con la responsabilidad social hacia los trabajadores, tratando al menos de minimizar a lo largo del tiempo y en comparación con otras empresas del sector, la ratio entre la remuneración media de los consejeros y la de los trabajadores. De nada sirve hablar de responsabilidad social corporativa en el ámbito laboral si una de las percepciones de equidad para los trabajadores, cual es la retribución salarial no está acompasada con la retribución de los otros grupos de interés de la empresa; en concreto, con la de los administradores.

Tal y como señalábamos anteriormente, si existe una generación de valor por parte de la empresa, parece obvio que ese valor ańadido distribuido sea tenido en cuenta para establecer el reparto del mismo entre todos los grupos de interés, incluidos los empleados.

\section{Análisis empírico}

\subsection{Objetivo y metodología}

Para explorar los diferentes aspectos relacionados con la información contable en la negociación colectiva de los convenios de empresa, hemos utilizado dos fuentes de datos principales:

- Una encuesta realizada a los directores de personal de diferentes empresas de Castilla y León, adheridos a la red de directores de recursos humanos de Castilla y León (CEDERED).

— Entrevistas a los tres sindicatos más representativos de la provincia de Burgos.

$\mathrm{Al}$ objeto de tener una primera aproximación de la realidad, realizamos un análisis de todos los Convenios Colectivos de empresa suscritos de una parte por los representantes de la empresa, y de otra, por la representación legal de los trabajadores, y presentados en la Oficina Territorial de Trabajo de Burgos. Este análisis supuso considerar a 69 empresas, que considerando todos los convenios colectivos publicados desde el año 2002 hasta el fin de 2014, abarca a más de 300 convenios colectivos de empresa. Los aspectos en los que prestamos más atención son los que tienen que ver con la forma de determinar la revisión sala- 
rial y la forma de determinar la participación en beneficios, si lo hubiera, al considerar que en estos puntos el uso de la información contable queda perfectamente justificado.

También con carácter previo al envío de los cuestionarios, concertamos entrevistas con negociadores de convenios colectivos tanto desde el punto de vista patronal como sindical, que permitieron cribar el cuestionario inicialmente previsto. Así, finalmente para cumplir con el objetivo propuesto, dividimos el cuestionario en las siguientes tres partes: una primera parte sobre negociación colectiva, una segunda sobre información económica y una tercera parte interrelacionando la información económica y la negociación colectiva.

Desde la otra perspectiva de la relación laboral, desarrollamos entrevistas en profundidad con los responsables provinciales de los tres sindicatos más representativos de la provincia de Burgos, tratando de ampliar nuestro conocimiento del punto de vista de los empleados y a la vez testar la opinión de los representantes de los trabajadores ante los resultados de la encuesta realizada previamente a los directores de recursos humanos.

El análisis realizado es eminentemente descriptivo, dada la naturaleza cualitativa de los datos resultantes de las encuestas y entrevistas realizadas.

\subsection{Resultados}

\subsubsection{Resultados de la encuesta a los directores de recursos humanos}

A través de CEDERED se enviaron vía correo electrónico 65 encuestas en el mes de mayo de 2015, habiendo cerrado la recepción de respuestas a finales del mes de junio de 2015. De las 65 encuestas enviadas, recibimos respuesta de 40 encuestas, lo que supone una tasa de respuesta del $62 \%$. Los resultados más representativos fueron los siguientes:

Los factores que consideran más importantes a la hora de la firma del convenio colectivo de empresa son los económicos; bien los de la situación económica de la empresa o bien la situación económica sectorial, relegando al último lugar la situación económica general. Jugando un papel también muy importante las relaciones empresa/sindicatos.

Las materias más determinantes en la negociación y en la discusión para la firma de los convenios colectivos de empresa son sin ninguna duda las que se refieren a la retribución fija y a los incrementos salariales. En segundo lugar también parece muy importante la negociación en lo referente a la jornada laboral, mientras que lo que se refiere a la retribución variable o productividad queda relegado a un segundo plano. 
Se pregunta a los directores de recursos humanos por la referencia que considerarían más aceptable para establecer la revisión salarial. La respuesta más valorada es la información económica de la propia empresa. Sigue siendo importante la referencia al IPC (Índice de Precios al Consumo) pero no al PIB (Producto Interior Bruto), factor que apenas es considerado.

La información económica de la empresa más relevante para fijar tanto el incremento salarial como los complementos variables es la información del momento de la negociación, aunque también es considerada la proyección de la información económica prevista para futuros ejercicios. (No es tomada apenas como referencia la información económica de años anteriores)

Para los directores de recursos humanos, la información contable es considerada poco por los representantes sindicales para modular las posiciones en la negociación. Un $60 \%$ de los encuestados manifiesta esa opinión, siendo una cifra ligeramente similar los que consideran que lo utilizan nada o mucho. A pesar de la respuesta anterior, consideran que la situación y los resultados de la empresa serían aceptados por un 77,50 \% para establecer las subidas salariales y las remuneraciones variables.

En la legislación laboral podemos encontrar cómo la situación económica negativa puede llevar a la inaplicación del convenio colectivo de empresa, y preguntamos a los responsables de recursos humanos si verían positivo que la legislación laboral incluyera, en el caso de situación económica positiva, a los trabajadores como partícipes de esta participación: son prácticamente la mitad los que se decantan por la inclusión y los que no lo hacen.

\subsubsection{Resultados de las entrevistas a los representantes sindicales}

Con un primer análisis de los resultados de la encuesta a los directivos de recursos humanos, establecimos reuniones con los responsables y expertos en negociación colectiva de los tres sindicatos más representativos en la provincia de Burgos: UGT, CCOO y USO. Esta entrevista nos permitió contrastar las opiniones empresariales con la percepción de los representantes de los trabajadores sobre aspectos clave de la información contable en la negociación colectiva, y la predisposición o no a aceptar las opiniones manifestadas por los directivos de recursos humanos.

En cuanto a los factores que consideran más importantes a la hora de la firma del convenio colectivo de empresa, coinciden totalmente con la opinión de la parte empresarial: son los económicos; bien los de la situación económica de la empresa o bien la situación económica sectorial, relegando al último lugar la situación económica general. No obstante, destaca el valor que para los sindicatos tiene el convenio sectorial, sobre todo según nos han manifestado en las entrevistas, porque juega como una especie de paraguas, en el sentido de que no 
siempre la situación económica de la empresa refleja la realidad de la explotación de la empresa. En especial manifiestan los sindicatos que en el caso de empresas que forman parte de un grupo, puede ocurrir que las empresas intenten demostrar una posición económica negativa de la empresa pero que en muchos casos viene motivada por la redirección de la facturación de la empresa hacia otras compañías que componen el grupo de empresas.

Las materias más determinantes en la negociación y en la discusión para la firma de los convenios colectivos de empresa son también las que se refieren a la retribución fija y a los incrementos salariales. Pero consideran aún más determinante la productividad y la retribución variable que lo referente a la jornada laboral y la flexibilidad.

La referencia que considerarían más aceptable para establecer la revisión salarial es el IPC. A pesar de que admiten la información económica de la empresa, ésta no genera demasiada confianza ni credibilidad por lo que buscan una referencia externa planteando también como alternativa la situación económica media del sector en el que se mueve la empresa. No consideran el PIB aunque fue planteado como alternativa válida dentro del proceso de discusión del III Acuerdo para la Negociación Colectiva.

Para los representantes sindicales, la información económica de la empresa más relevante a la hora de fijar tanto el incremento salarial como los complementos variables es toda la información disponible tanto anterior, del momento de la negociación, como futura. No quieren en absoluto prescindir de la información económica anterior.

La información contable es considerada poco o nada para modular las posiciones en la negociación, síntoma de la desconfianza hacia la misma ya que sí que consideran a la información económica como factor determinante en la negociación. A pesar de la respuesta anterior, consideran que la situación y los resultados de la empresa sí serían aceptados para establecer las subidas salariales y las remuneraciones variables. Los representantes sindicales abogan, sin ninguna duda, porque en la legislación laboral se incorporara a los trabajadores como partícipes de la situación económica positiva de la empresa.

\subsection{Discusión de los resultados}

De los resultados anteriores obtenemos evidencias de que ambas partes consideran que los factores más importantes a la hora de la firma del convenio colectivo de empresa son los económicos. Este hecho constata la importancia de la información económica, ya que dentro de los datos económicos se tiene en cuenta sobre todo los propios de la situación económica de la empresa (incluso la situación económica del grupo de empresas, en el caso de formar parte de alguno). Así, consideramos que en este caso se tendría que incorporar la información contable del 
grupo empresarial para hacer partícipes también a los empleados, en ciertos casos, de la rentabilidad global del conglomerado. También destacamos la percepción, por parte de los representantes de los trabajadores, en el sentido de que las empresas muestran peores cifras en momentos previos a la negociación, cifras que mejoran en cuanto se produce la firma de los convenios colectivos de la empresa.

Las materias más determinantes en la negociación son para ambas partes las que hacen referencia a las diferentes formas de retribución. Evidentemente las exigencias y posibilidades retributivas tienen que ver con la situación económica de la empresa.

En el marco de la revisión salarial, las posturas están claramente enfrentadas. Frente a los directores de recursos humanos que abogan por la situación económica de la empresa; los representantes sindicales optan por el Índice de Precios al Consumo. La información económica de la empresa y concretamente la contable siempre se la ha considerado como referencia válida en las relaciones laborales y evidentemente confirma la necesidad de transmisión de información contable. Aunque la reticencia sindical no viene tanto por la negación de la validez de la información contable en sí, sino por la falta de confianza y credibilidad hacia la misma.

La información económica de la empresa a considerar por ambas partes es la más amplia posible, ya que los directores de recursos humanos plantean la información presente y futura; a la que los sindicatos ańaden la información pasada. La propia negociación que en muchos de los casos finaliza en un convenio de empresa con una vigencia superior al año natural puede favorecer el uso de esta información previsional. Circunstancia que consideramos no está prevista adecuadamente en la legislación laboral al no haber ninguna referencia expresa en el ET a la transmisión de información contable prospectiva.

A pesar de la importancia que ambas partes dan a la información contable, consideran que ésta no se utiliza expresamente para modificar las posiciones negociadoras, síntoma de desconfianza en la misma por parte de los representantes sindicales. Aun con esa desconfianza, sí lo valoran dentro de las revisiones salariales y remuneraciones variables, lo que evidencia el carácter preferente de la información contable.

Ante la posibilidad de regular la obligatoriedad de dar participación en beneficios a todos los trabajadores cuando la situación económica empresarial lo permita, los directores de recursos humanos no lo consideran en su mayoría, mientras que los representantes sindicales lo verían muy razonable. No parece muy consecuente esta interpretación desde el punto de vista empresarial; si se tiende a remuneración variable, parece que lo recomendable sería en todos los sentidos. Mucho más consecuente es la postura sindical, ya que el reconocimiento de la situación económica de la empresa lo sería tanto para sufrir la situación económica negativa como para participar en beneficios. 


\section{Conclusiones}

En sintonía con las evidencias de la literatura anterior relativa a la Teoría de la agencia y la Teoría de los contratos, existe una divergencia absoluta de intereses. De las encuestas a directivos de recursos humanos de Castilla y León y entrevistas a los representantes de los sindicatos mayoritarios de la provincia de Burgos hemos obtenido como resultados que las empresas con circunstancias económicas negativas son propensas a poner de manifiesto y utilizar la información contable. Sin embargo, no se observa este comportamiento cuando la situación económica es positiva. Mientras que los trabajadores, a pesar de las dudas que plantean ante la información económica de las empresas, sí estarían dispuestos a considerarla en cualquier circunstancia, sobre todo la información contable interna, y especialmente si se les diera participación en los resultados positivos. Esta actitud parece más apropiada para incentivar comportamientos que permitan la consecución de objetivos comunes para las partes.

Por otra parte, consideramos interesante que las empresas suministren a los representantes de los trabajadores, con carácter periódico, certificado de estar al corriente de las obligaciones fiscales; ya que hemos podido constatar que la administración tributaria, a pesar de ser acreedora de gran número de empresas, por importes muy considerables, apenas insta los concursos de acreedores necesarios. Sin entrar a considerar, si es o no una actuación negligente y por tanto "cómplice» y de alguna manera responsable también de la situación de insolvencia de la empresa, el conocimiento por parte de los representantes de los trabajadores de esta información sería de suma importancia para establecer la estrategia negociadora.

El hecho de haber realizado la encuesta a directores de recursos humanos de Castilla y León, junto con las entrevistas a representantes sindicales de la provincia de Burgos, supone disponer de una muestra representativa de empresas de diferentes sectores y tamaños. Sin embargo, una de las principales limitaciones consiste en que el ámbito de actuación es local, aun cuando consideramos que gran parte de estas respuestas podrían ser extrapolables al resto del estado.

Así, futuras investigaciones podrían contemplar, entre otros, los siguientes objetivos:

- Comparar las opiniones recogidas en este trabajo con las de directores de recursos humanos y representantes sindicales de otras provincias, para tratar de extrapolarlo al conjunto del estado español.

- Analizar la variación de las remuneraciones de los administradores en empresas cotizadas con la remuneración de los trabajadores regulados por convenio colectivo empresarial. 
- Analizar la posible relación entre mejora de productividad y participación variable en resultados para todos los trabajadores.

- Intensificar mediante el estudio del caso el análisis causa - efecto de alguna empresa que ha pasado de no utilizar información contable en la negociación colectiva a sí utilizarla.

En todo caso, creemos que este estudio ha ampliado las evidencias sobre el uso de la información económica en la negociación colectiva. Estamos convencidos que el aumento de esa transmisión de información podría facilitar a los representantes sindicales el conocimiento de la realidad empresarial, agilizando así el encuentro de las partes en la negociación de los convenios colectivos de empresa. En resumen, consideramos que la información económica es muy importante para la negociación colectiva. Las empresas deberían estar obligadas, bien por ley o por interpretación amplia del ET, a transmitir todo tipo de información contable a los representantes de los trabajadores; concretamente tal y como hemos apuntado a lo largo del trabajo: las cuentas anuales del último ejercicio cerrado, balance de comprobación a la fecha más próxima a la negociación, información económica previsional, información procedente de la contabilidad analítica (productividad, nivel de actividad, etc.), información de carácter fiscal, información económica sectorial, información sobre las remuneraciones de los administradores, ... y en todos los casos, referenciado tanto a la empresa individual como a las cuentas consolidadas, en el caso de formar parte de un grupo de empresas.

\section{Bibliografía}

Amernic, J. (1983): «Accounting for collective bargaining», CGA Magazine, enero, 28-30.

Amernic, J. (1985): «The roles of accounting in collective bargaining», Accounting, Organizations and Society, 10, 2, 227-253.

Amernic, J. Y Craig, R. (1992): «Employer equivocality and union heterogeneity as determinants of the role of accounting in collective bargaining», Accounting, Auditing \& Accountability Journal, 5, 1, 60-79.

Aragon Medina, J.; Rocha Sánchez, F. y De la Fuente Sanz, Luis (2011): «La negociación colectiva y la información económica en España», Estudios de la Fundación, área de empleo y relaciones laborales, 43, 46-57.

Barbash, J. (1979): "Collective Bargaining and the Theory of Conflict», Relations industrielles / Industrial Relations, 34, 4, 646-659.

Bougen, P. (1989): "The emergence, roles and consequences of an accounting-industrial relations interaction", Accounting, Organizations and Society, 14, 3, 203-234.

Brio Gonzalez, E. Del (1995): «La responsabilidad social de la contabilidad», Técnica Contable., 801-818.

Conse, R. (1937): «The nature of the firm», Economica, 4, 386-405.

Cooper, D. y Essex, S. (1977): "Accounting information and employee decision making», Accounting, Organizations and Society, 2, 3, 201-217. 
CoOper, D. y SHerer, M. (1984): «The value of corporate accounting reports: arguments for a political economy of accounting", Accounting, Organizations and Society, 9, 3/4, 207-232.

Craft, J. (1981): «Information disclosure and the role of the accountant in collective bargaining», Accounting, Organizations and Society, 6, 1, 97-107.

Culliman, C.; Clark, M. y Kniblett, J. (1994): "Accounting information and collective bargaining: a literature review and research framework», Journal of Accounting Literature, 13, 44-80.

Cuthbert, N.H. y Whitaker, A. (1977): «Disclosure of information and collective bargaining: a re-examination", Journal of Business Finance \& Accounting, 4,3, 373-378.

Fama, E. (1980): "Agency problems and the theory of the firm», Journal of Political Economy, 88, 288-307.

Foley, B.J. y Maunders, K.T. (1977): «Accounting information disclosure and collective bargaining», New York; Holmer and Meier Publishers.

Fox, A. (1974): «Beyond Contract: Work, Power and Trust Relationships», Faber and Faber, London.

Gibbins, M.; Richardson, A. y Waterhouse, J. (1990): «The management of corporate financial disclosure: opportunism, ritualism, policies and process», Journal of Accounting Research, 28, 1, 121-143.

Goggans, T. (1964): «The accountant's role in wage negotiations», The Accounting Review, Julio, 627-630.

Gold, M. Levie, H. Y Moore, R. (1979): «The shop steward's guide to use of company information", Spokeman Books

Gospel, H. (1978): «The disclosure of information to trade unions: approaches and problems», Industrial Relations Journal, 9, 3, 18-26.

Hart, O. (1983): «Optimal labour contracts under asymmetric information: an introduction", Review of Economics Studies, 50, 3-35.

Hicks, J.R. (1963): «The theory of wages», New York. St Martin Press.

Hopwood, A. (1985): «The tale of a committee that never reported: disagreements on interwining accounting with the social», Accounting, Organizations and Society, 10, 3, 361-377.

Jackson-Cox,J.; Thirkell, J. y Mc Queeney, J. (1984):»The disclosure of company information to trade unions: the relevance of the acas code of practice on disclosure», Accounting, Organizations and Society, 9, 3/4, 253-273.

Jensen, M. y Meckling, W. (1976): "Theory of the firm: managerial behavior, agency costs, and ownership structure», The Journal of Financial Economics, 3, 305-360.

Kleiner, M. (1984): «Public policy implications of financial information requirements under the National Labor Relations Act», Journal of Accounting and Public Policy, 3, Winter, 253-257.

Knights, D. y Collinson, D. (1987): «Disciplining the shop floor. A comparison of the disciplinary effects of managerial psychology and financial accounting», Accounting, Organizations and society, 12, 5, 457-477.

LAFFARGA Briones, J. (1993): «Información contable y negociación colectiva», Trabajo de Investigación. Universidad de Cádiz.

LewIs, N.R.; Parker, L.D. Y Sutcliffe, P.(1984): «Financial reporting to employees: the pattern of development 1919 to 1979», Accounting, Organizations and Society, 9, 3/4, 275-289.

Liberty, S. y Zimmerman, J. (1986): «Labor union contract negotiations and accounting choices», The Accounting Review, LXI, 4, 692-712. 
Maunders, K. (1981): «Accounting information and industrial relations: towards a managerial evaluation of the disclosure policy decision», Managerial Finance, 7, 2, 2-5.

Maunders, K. (1984): «Employment reporting. An investigation of user needs, measurement and reporting issues and practice», The Institute of Chartered Accountants in England and Wales. Londres.

Maunders, K. y Foley, B. (1984): "Information disclosure and the role of the accountant in collective bargaining-some comments", Accounting, Organizations and Society, 9, 1, 99-106.

Mitchell, F.; Sams, K.; Tweedie, D. y White, P. (1980): «Disclosure of information: some evidence from case studies», Industrial Relations Journal, 11, 5, 53-62.

OAKEs, L. y CovAlEski, M. (1994): «A historical examination of the use of accountingbased incentive plans in the structuring of labor-management relations», Accounting, Organizations and Society, 19, 7, 579-599.

Ogden, S. y Bougen, P. (1985): «A radical perspective on the disclosure of accounting information to trade unions", Accounting, Organizations and Society, 10, 2, 211-224.

Ogden, S. (1992): «The limits to employee involvement: profit sharing and disclosure of information", Journal of Management Studies, 29, 2, 229-248.

Owen, D.L. y Lloyd, A. (1985): «The use of financial information by trade union negotiators in plant level collective bargaining», Accounting, Organizations and Society, 10, 3, 329-350.

PacitTi, A. (1990): «Financial information for employees», Paper presented at the European Accounting Association Annual Congress. Budapest. Abril.

Peel, P.F. y Pope, D.A (1981): «Information disclosure to employees and rational expectations", Journal of Business Finance and Accounting, 8, 139-142.

Pillsbury, W.F. (1958): «Organized labor's view of corporate financial information», Journal of Accountancy, June, 46-56.

Pons Felix, A. (1996): «Información y relaciones laborales», Técnica contable, 59-68.

Propuesta de Directiva del Parlamento Europeo y del Consejo de 9 de abril de 2014, por la que se modifica la Directiva 2007/37/CE

Rahman, M. y Mccosh, A. (1976): «The influence of organisational and personal factors on the use of accounting information: an empirical study", Accounting, Organizations and Society, 1, 4, 339-355.

Resolución de 15 de junio de 2015, de la Dirección General de Empleo, por la que se registra y publica el III Acuerdo para el Empleo y la Negociación Colectiva 2015, 2016 y 2017.

Ross, S. A. (1973): «The economic theory of agency: the principal's problem», American Economic Review, 63, 2, 134-139.

Roth, A. y Murnighan, J. K. (1982): «The role of information in bargaining: an experimental study», Econometrica, 50, 5, 1123-1142.

Salamon y Smith (1979): "Corporate Control and Managerial Misrepresentation of Firm Performance», The Bell Journal of Economics, 10, 1, 319-328.

Sherer, M.; Soutworth, A. y Turley, S. (1981): "An empirical investigation of disclosure usage and usefulness of corporate accounting information", Managerial Finance, 7, 2, 6-11.

Simon, H. (1951): "A formal theory of the employments relationship», Econometrica, 19, 293-305. 
TомсzYк, S.H. (1975): Financial ratios and the ability to pay wage increases, DBA dissertation. University of Kentucky.

Tracy, L. y Peterson, R. (1986): "A behavioral theory of labor negotiations- how well has it aged", Negotiation Journal, Enero, 93-108.

Walton, R. y Mckersie, R. (1965): A behavioral theory of labornegotiations, McGrawHill, New York.

Yamaji, H. (1986): «Collective bargaining and accounting disclosure: an inquiry into the changes in accounting policy", International Journal of Accounting, 22, 11-23. 